\title{
Robust active vision industrial CAD parts recognition system
}

\author{
Tushar Jain* and Meenu \\ Mechanical Engineering Department, \\ NIT Kurukshetra, India \\ Email: tusharkaid@gmail.com \\ Email: meenu_1625@ymail.com \\ *Corresponding author
}

\author{
H.K. Sardana \\ CSIO, \\ Chandigarh, India \\ Email: hk_sardana@hotmail.com
}

\begin{abstract}
In automated assembly systems the machine parts identification is entirely different from simple object recognition; moreover the ability of humans to differentiate between correct and not correct machine parts is better but it is a difficult task for a machine. In general, with fast moving machine parts on the conveyor manual defect detection by human inspectors is impractical. Also it is expensive, inaccurate, subjective, eye straining and causes other health issues to quality control inspectors. A computer vision-based non-contact inspection technique is developed with image processing methods by considering these problems, for defect detection in industrial machine parts. The present work will help the industrial robot used in assembly process and industrial inspection systems. In this paper features-based industrial object detection techniques are implemented in MATLAB to recognise the presence of the industrial CAD parts in the query image. In the end the actual industrial tool images are also used to show the accuracy and robustness of the proposed machine vision system for industrial manufacturing automation.
\end{abstract}

Keywords: active vision; industrial CAD parts; image processing; parts recognition; feature-based algorithms; intelligent systems; robotics.

Reference to this paper should be made as follows: Jain, T., Meenu and Sardana, H.K. (2018) 'Robust active vision industrial CAD parts recognition system', Int. J. Intelligent Machines and Robotics, Vol. 1, No. 1, pp.16-33.

Biographical notes: Tushar Jain is a $\mathrm{PhD}$ student in Mechanical Engineering at National Institute of Technology Kurukshetra India. His primary research interests are machine vision, image processing and computational intelligence.

Meenu is an Associate Professor in the Department of Mechanical Engineering at National Institute of Technology Kurukshetra India. Her research focuses on machine vision (image processing, image analysis, pattern recognition), artificial neural network, genetic algorithm, taguchi technique, and response surface methodology. 
H.K. Sardana is a Chief Scientist at Central Scientific Instrument Organization Chandigarh India. His research focuses on pattern recognition, computer vision and medical image analysis.

\section{Introduction}

Today in various sorts of item assembling enterprises (e.g., locks, adapt boxes, wake up timers, motors and engines and so forth.) and bundling businesses with extensive scale generation units robotised get together frameworks are broadly utilised. They utilised automated gadgets, for example, picture catching unit, transport, part acknowledgment unit, part feeder, part determination unit, and canny robots that take after settled grouping of ventures to amass the item. The productivity of general item assembling and assessment process can be expanded with modern robotisation and it additionally limits assets and spares time. The image-based systems still have open issues in order to meet the latest manufacturing requirements for simplicity, low cost as well as the limited maintenance requirements. In this direction, there is a method proposed for the recognition of $3 \mathrm{D}$ randomly placed objects for eventual robotic handling. The method includes a $2 \mathrm{D}$ vision system and is combined with data from computer-aided design (CAD) files for the generation of 3D coordinates. It is generic and can be used for the identification of multiple randomly placed objects.

Figure 1 A schematic diagram for machine parts detection in model assembly line

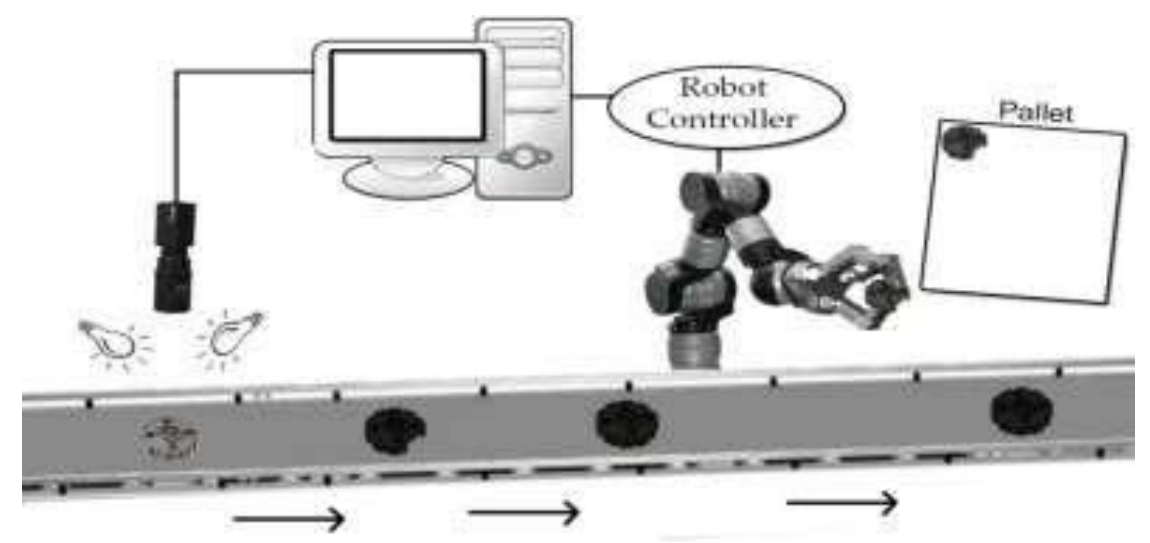

Imperative parts of vision frameworks, in mechanical applications, are the straightforwardness of the calculation, the minimal effort and the lessened requirement for upkeep, while viewpoints, for example, the quick and powerful identification still constitutes an unsolved issue. Despite the fact that enough productive and precise calculations have been created, the processing speed still neglects to meet the present day fabricating necessities (Kodagali and Balaji, 2012). The issue turns out to be further muddled inferable from the articles' properties, for example, shape, material, shading, and so on. Moreover, the necessities for straightforwardness and ease are specifically 
associated with the creation rate that is required to be expanded with the presentation of automated gear in cutting edge generation lines (Chryssolouris, 2006). By and large regarding profitability there are three classes of get together frameworks to be specific low, medium and high volume generation units. The congregations frameworks are completely mechanised in high volume creation, get together of parts in other two classes are performed in semi-computerised or manual by hand. The cost for setting up such frameworks at first is high, yet in longer run it spares time, cash and work. The advantages of such framework are tremendous amount of creation, stable item plan with great quality and unwavering quality. Figure 1 shows mechanical production system with machine parts recognition framework.

\section{Designing and rendering process}

In this segment CAD parts configuration is clarified. Section 2.1 portrays the outline of Industrial CAD parts utilising Solid Edge ST8 and wire outline demonstrating indicating rendering utilising standard tessellation language (STL) is talked about in Section 2.2 that aides in making the database of various Industrial CAD parts. This paper introduces a computationally proficient 3D PC vision-based way to deal with perceive the Mechanical CAD parts.

\subsection{Industrial CAD parts design}

In the present research work the mechanical CAD parts are outlined with Solid Edge ST8. The outline is made by 2D drawings to 3D question. Since the Solid Edge ST8 underpins an immediate collaboration with the planner, thus the need to compose program is not required at all and we can without much of a stretch discover the distinctive surfaces. Strong Edge is a blend of various programming devices that envelops all means of the item outline and advancement procedures like 3D sections creation, plan re-enactment, parts assembling and configuration parts administration. Strong Edge gives easy to use adaptable interface to coordinate the speed with straightforwardness utilising synchronous innovation for the control of parametric outline. Figure 2 demonstrates the distinctive Industrial CAD parts outlined utilising Solid Edge ST8.

Figure 2 Diverse mechanical CAD parts designed using Solid Edge ST8 (see online version for colours)
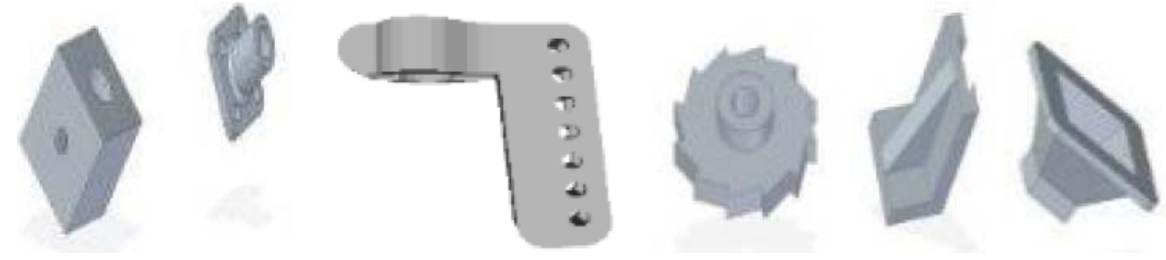

\subsection{CAD parts rendering using STL}

The STL remains for standard tessellation language. All propelled CAD programming underpins STL document organise. The major mechanical use of it is in CAD/CAM, fast prototyping machines and $3 \mathrm{D}$ printing. In rapid prototyping machines the STL document 
configuration is utilised to exchange CAD information. Triangular work is produced on the surface of CAD model by utilising this arrangement.

In CAM environment the triangulated geometry is foreign made utilising STL. At long last this data helps in creating and assembling blunder free modern items. STL documents demonstrate the geometrical surface of a 3D CAD show. The data about different qualities of CAD model like surface and shading are not spoken to with this arrangement. The CAD question rendering STL organise yield is appeared in Figure 3 utilising MATLAB.

Figure 3 MATLAB rendering of mechanical CAD parts (see online version for colours)

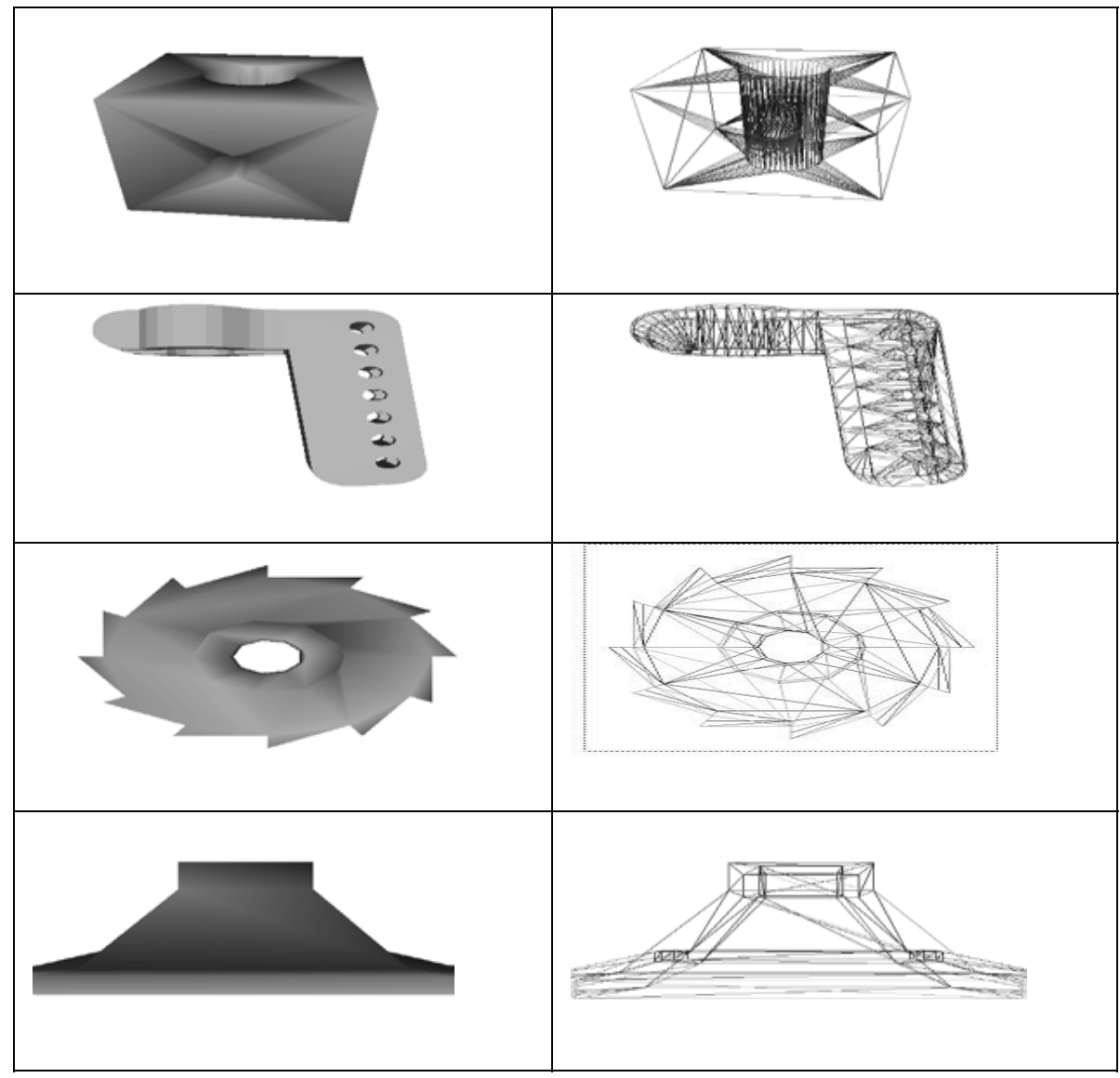

\section{Literature review}

The requirement for objects recognition system is met in various modern applications, where diverse objects of variable shapes and sizes ought to be dealt with. Point by point audits on the most recent sensor innovations and the exploration patterns for get together frameworks have demonstrated that vision systems are reasonable for objects recognition, review and robot taking care of utilisations (Santochi and Dini, 1998; Janocha and Seidel, 
1988; Michalos et al., 2012; Vahrenkamp et al., 2009; Han et al., 2000, 2001). Investigation has been done in the plan parts of the machine vision frameworks for modern applications (Golnabi and Asadpour, 2007), and has prompted to enhancements in dependability and item quality (Kodagali and Balaji, 2012; Beserra Gomes et al., 2013; Xie et al., 2008).

The speeded-up robust features (SURF) (Bay et al., 2006) and scale invariant feature transform (SIFT) (Li and Ma, 2009) descriptors extracts a specific object in an occluded objects image or to detect exact matching between two images. The features extracted corresponding to a specific object in an image must be invariant to rotation and scaling for perfect matching and recognition (Mikolajczyk and Schmid, 2001). In present scenario these techniques are applied to real time object recognition, object matching and inspection, query image retrieval etc. SIFT (Lowe, 2004) is widely used in different machine vision applications, but at the cost of computationally expensive SIFT feature points calculation for the given object. In contrast to this SURF descriptors are comparatively more computationally efficient in comparison to SIFT (Mortensen et al., 2005). That's why machine vision application areas for object recognition prefers SURF over SIFT. These approaches have been used to extract particular invariant components from images that can be utilised to perform solid coordinating between various perspectives of an object (Panchal et al., 2013).

SURF is also used as an extraordinary algorithm with illumination and rotation invariant features for traffic sign detection (Zhao et al., 2013). The detection of mechanical parts features like lines, circles, angles etc. using the Hough transform is described efficiently (Alshennawy, 2014). The industrial application of this approach is to detect mechanical parts having holes. The object pose estimation based on features using Hough is also shown (Seib et al., 2014). A new descriptor named maximally stable extremal regions (MSER) features extracts regions with uniform intensity and also shows independency about scale (Matas et al., 2002). SURF, MSER, Eigen, FAST and Harris features-based algorithms (Harris and Stephens, 1988) are also used on perfect matching of different objects in many applications of computer vision. This approach extracts unique features and helps in detecting the object image (Farman et al., 2016).

There is a technique proposed for the recognition of 3D arbitrarily set items for inevitable automated dealing with. The technique incorporates a $2 \mathrm{D}$ vision system and is joined with information from PC helped plan (CAD) documents for the era of 3D directions. It is non-specific and can be utilised for the distinguishing proof of different arbitrarily set items (Tsarouchi et al., 2016). The object recognition technique has in like way been analysed in Pham and Smeulders (2005), Bai et al. (2008), Shokoufandeh et al. (1999), Frome et al. (2004), Laptev (2009) and Melkemi and Djebali (2001), while 3D vision structures have been appeared in Bicego et al. (2005), Scovanner et al. (2007) and Moreels and Perona (2007). Such structures have the upsides of seeing the things' qualities, regardless they rely on upon baffled figuring's and are provoke to frustrations in mechanical conditions. In many situations these feature types shown better results (Mikolajczyk and Schmid, 2005; Mikolajczyk et al., 2005; Schmid et al., 2000).

\section{Proposed approach}

A non-contact machine vision-based CAD parts recognition system is proposed. The sequence of steps followed in the proposed system is: image acquisition, image 
pre-processing, shape extraction, features extraction, comparability coordinating and CAD machine part recognition. These steps are summarised in flow diagram in Figure 4.

Figure 4 A flow diagram for the proposed industrial CAD parts recognition system

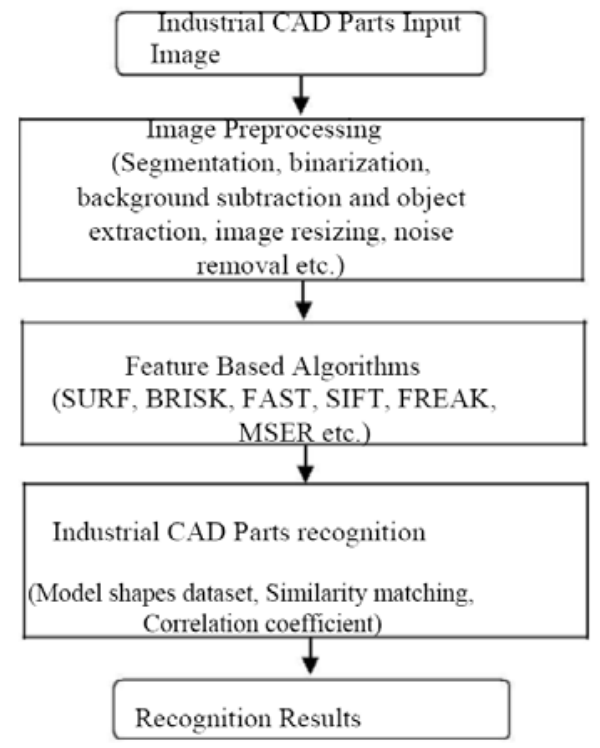

The image acquisition framework gets information from the moving machine parts in line which is a piece of the mechanical production system setup. To change the information reasonable for further handling the caught machine CAD part image information experiences image pre-processing methods like binarisation, noise removal, background subtraction and so forth to change the information appropriate for further processing. At that point a shape of the machine parts are extricated and standardised by equivalent part territory strategy to depict the elements of the parts. It gives vital pieces of information for machine part shape acknowledgment. For test reason a model shape for every machine part is created in CAD software Solid Edge, the parts recognition is performed with just reference to the model shape.

\section{Experimental results}

The exploratory outcomes utilising SURF, SIFT, BRISK, FREAK, FAST and MSER approach of modern CAD parts recognition are introduced in this area with relative proposed approach. Figure 5(a) and Figure 5(b) demonstrates the mechanical CAD parts first image and second image separately with scaling and rotation of first image so that these two images are analysed for CAD parts recognition. Figure 5(c) and Figure 5(d) speaks to the putatively coordinated utilising SURF, BRISK and FREAK. Figure 5(e) speaks to the last CAD question distinguished image utilising proposed strategy invariant to scaling and turn that might be the states of real mechanical framework. 
Figure 5 Industrial CAD part 1 results using SURF, BRISK and FREAK (see online version for colours)

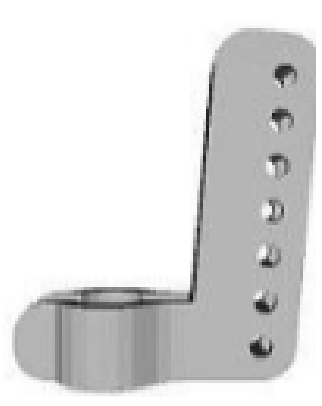

(a)

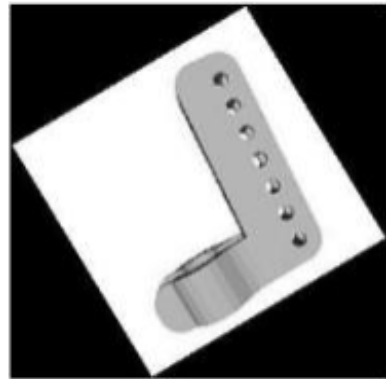

(b)

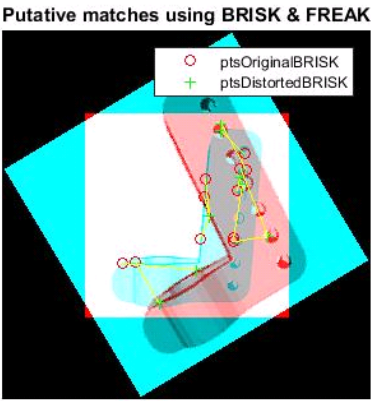

(c)

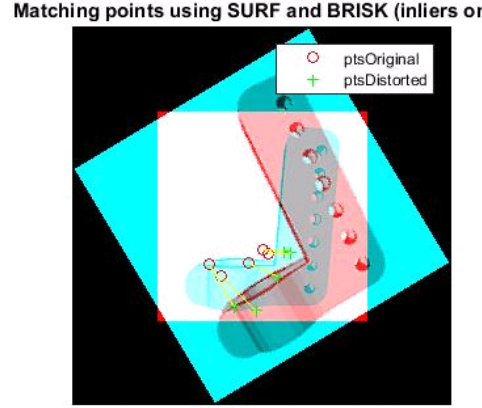

(d)

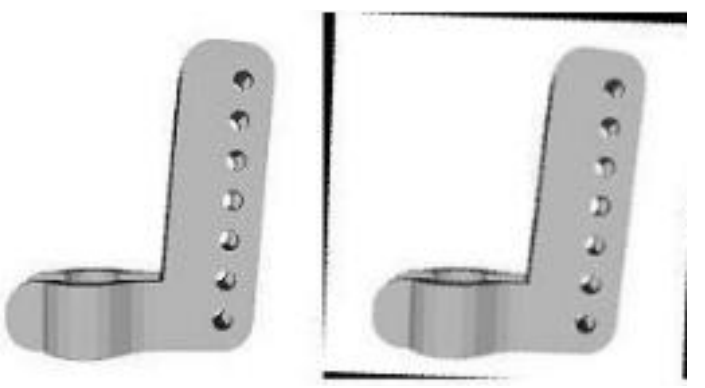

(e)

Figure 6 Industrial CAD part 2 results using SURF, BRISK and FREAK (see online version for colours)

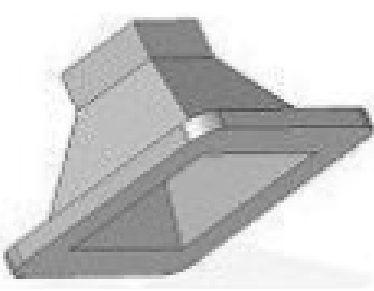

(a)

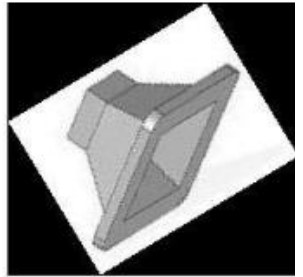

(b)

Putative matches using BRISK \& FREAK

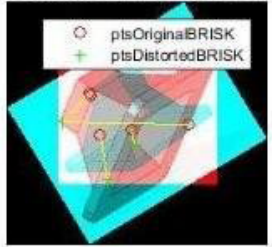

(c)

Uatching points using SURF and BRISK (inliers only

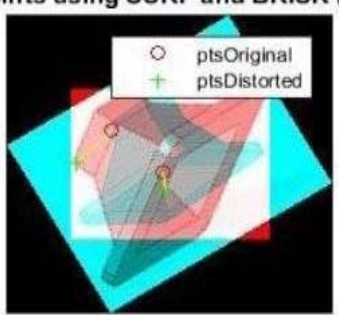

(d)

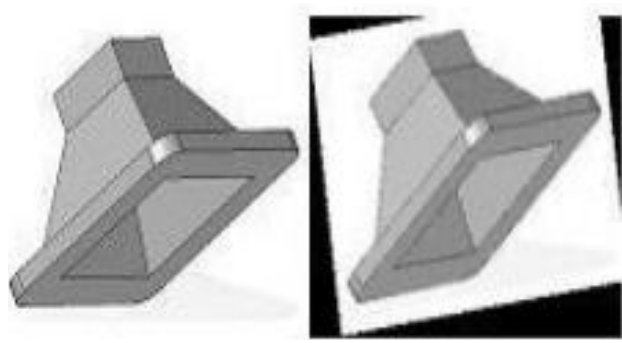

(e) 
Figure 7 Industrial CAD part 3 results using SURF, BRISK and FREAK (see online version for colours)

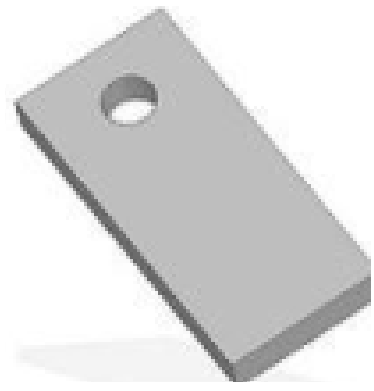

(a)

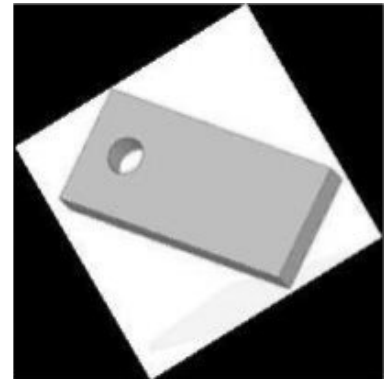

(b)

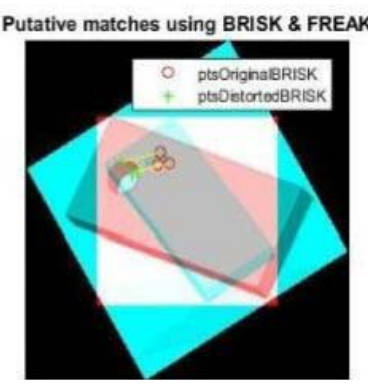

(c)

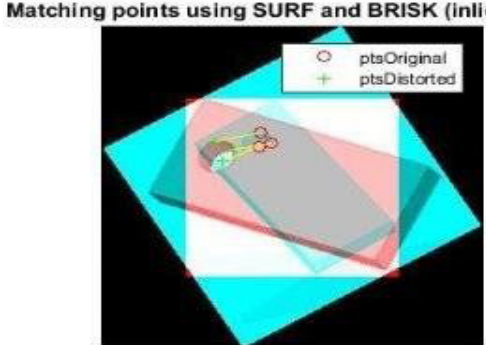

(d)

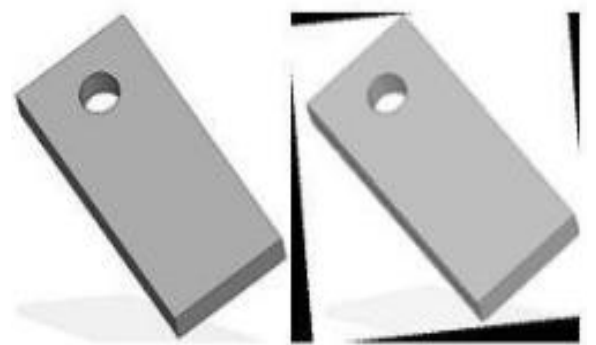

(e)

Figure 8 Industrial machine tool 1 results using SURF, BRISK and FREAK (see online version for colours)

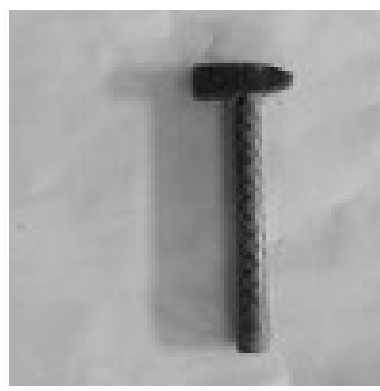

(a)

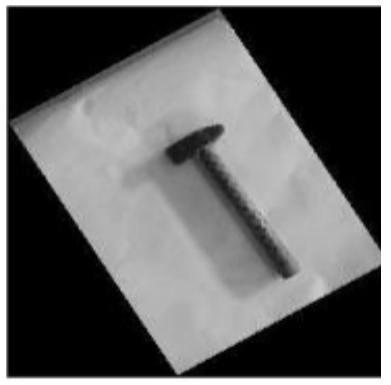

(b)

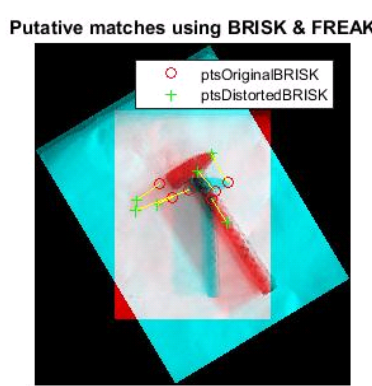

(c)

Matching points using SURF and BRISK (inliers only)

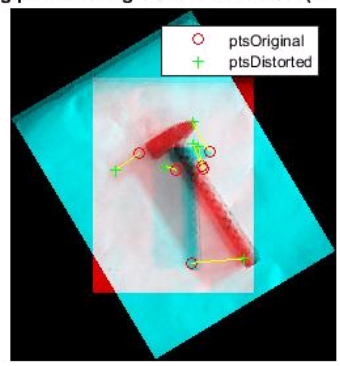

(d)

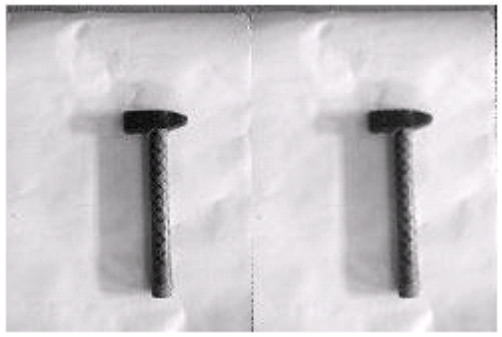

(e) 
Figure 6 and Figure 7 shows the similar kind of results with different CAD parts using SURF, BRISK and FREAK techniques. As it is clear that the 3D parts are recognised with accuracy. In Figure 8(a) hammer is taken as an input image and Figure 8(b) shows the hammer image after rotation and scaling. Figure $8(\mathrm{c})$ represents the putatively matching using BRISK and FREAK while Figure 8(d) shows matching points using SURF and BRISK.

Figure 9 Industrial machine tool 2 results using SURF, BRISK and FREAK (see online version for colours)

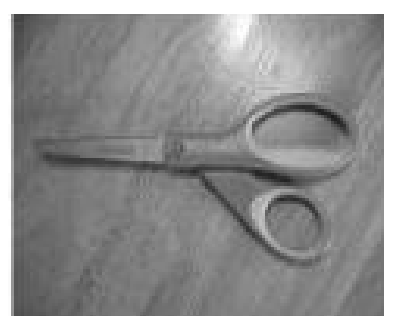

(a)

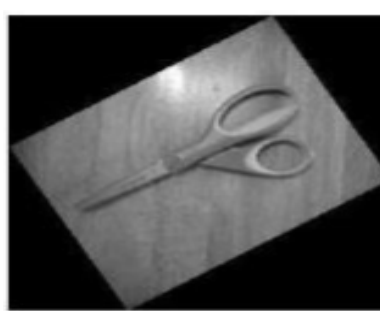

(b)

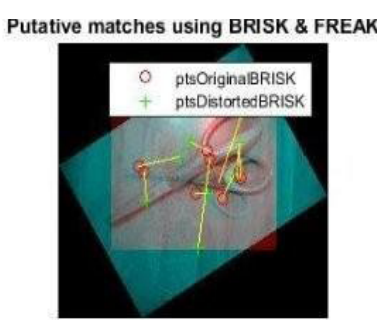

(c)

Matching points using SURF and BRISK (inliers only)

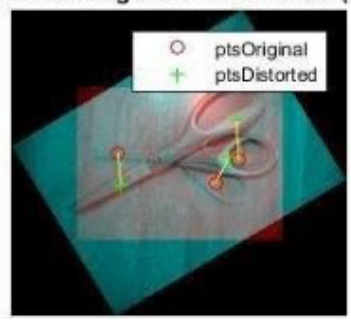

(d)

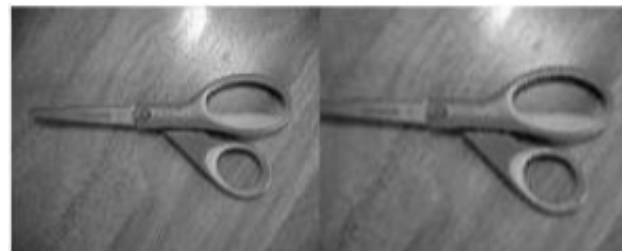

(e)

The final output showing the perfect matching of hammer which is a real mechanical industrial object in Figure 8(e). Figure 9 shows the image of scissor in real environment to be recognised with same approach. Figure 10 and Figure 11 depicts the successful implementation of proposed approach on other real tools images. Hence the experimental results show that the proposed approach is doing well on 3D CAD parts as well as on real world industrial tools. The final results of industrial machine tools using SIFT is shown in Figure 12 to Figure 14 while Figure 15 shows CAD part results. The different outputs show the accuracy of method for different tools in 2D as well as 3D orientations. The different machine tools selected for SIFT are saw, scissor and hammer in various positions. Figure 16 shows the final results of 3D CAD part using MSER. The object is rotated and then scaled with certain value to show the invariant characteristics of this technique. Similarly Figure 17 shows the recognition results of hack saw with other tools present in the same image like hammer, saw, file etc. Hence it is clear with this example that this method is capable of recognising correctly the query object image. Figure 18 is a variation in this technique. Here variety of hammers is taken as an object. This case shows the capability of MSER approach to detect a specific object with similar kinds of objects. Hence it is clear that with MSER we can detect objects with more accuracy and efficiency and it can be a good method for industrial environment. Figure 19 and Figure 20 shows the results of different objects with the same approach. 
Figure 10 Industrial machine tool 3 results using SURF, BRISK and FREAK (see online version for colours)

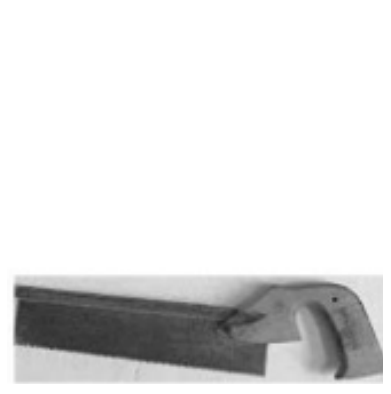

(a)

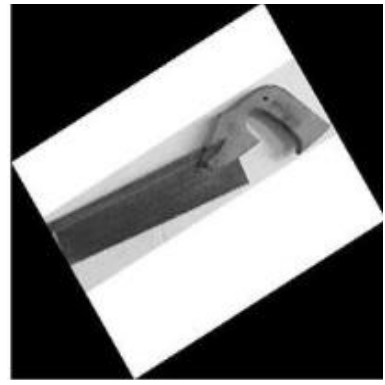

(b)

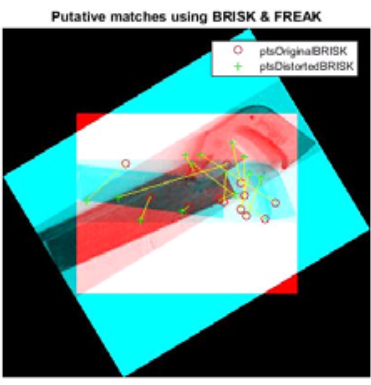

(c)

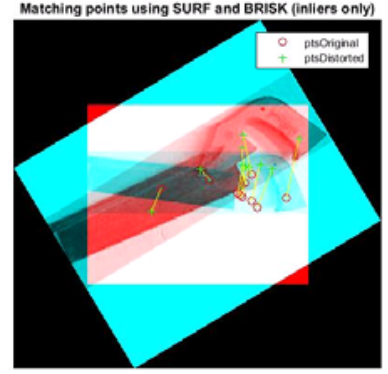

(d)

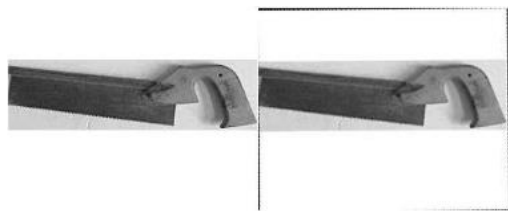

(e)

Figure 11 Industrial machine tool 4 results using SURF, BRISK and FREAK (see online version for colours)

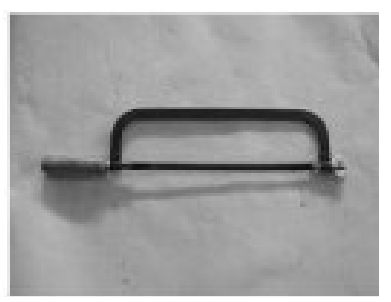

(a)

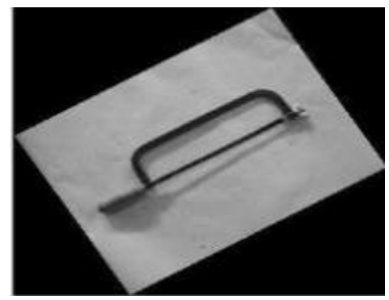

(b)

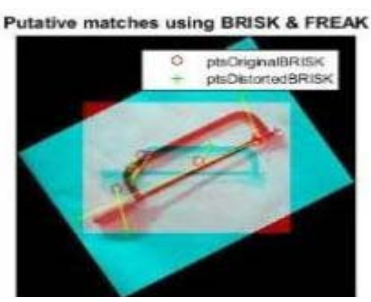

(c)

Matching points using SURF and BRISK (inliers only)

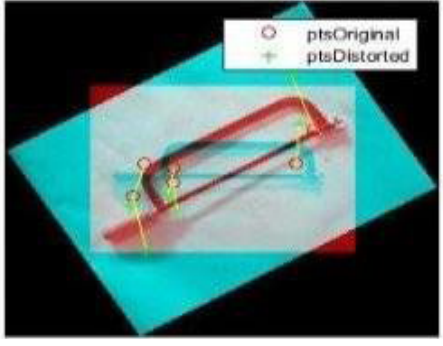

(d)

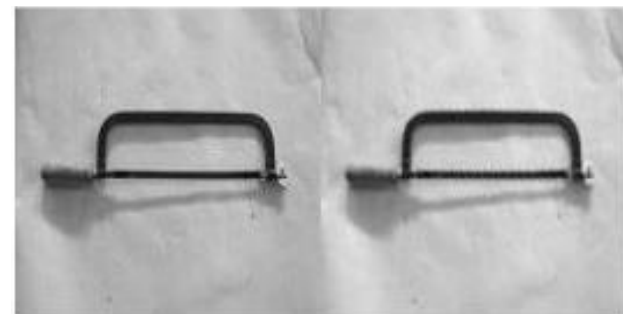

(e) 
Figure 12 Industrial machine tool 4 results using SIFT (see online version for colours)
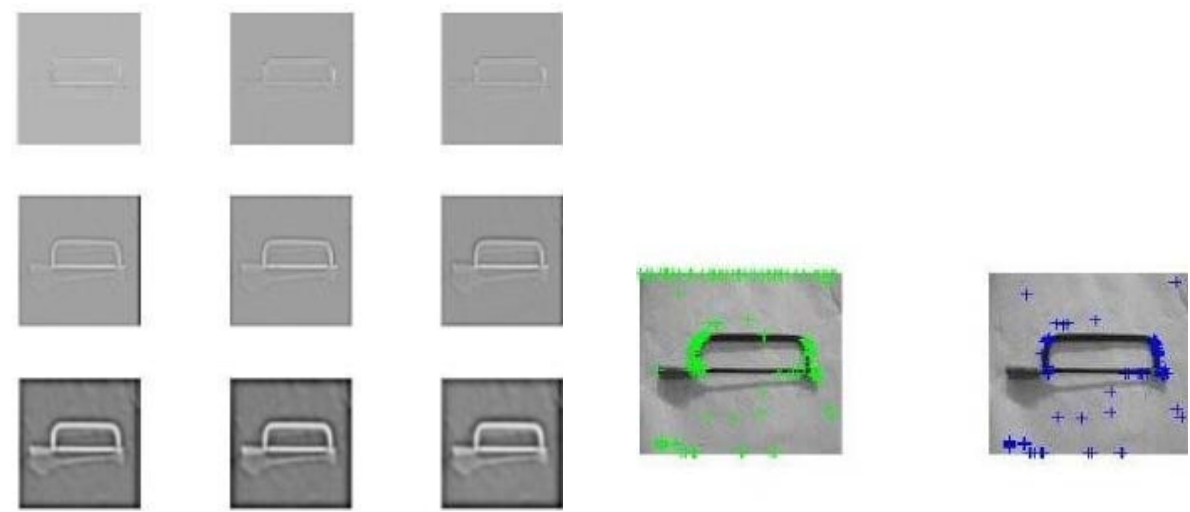

(a)

(b)

Figure 13 Industrial machine Tool 2 results using SIFT (see online version for colours)
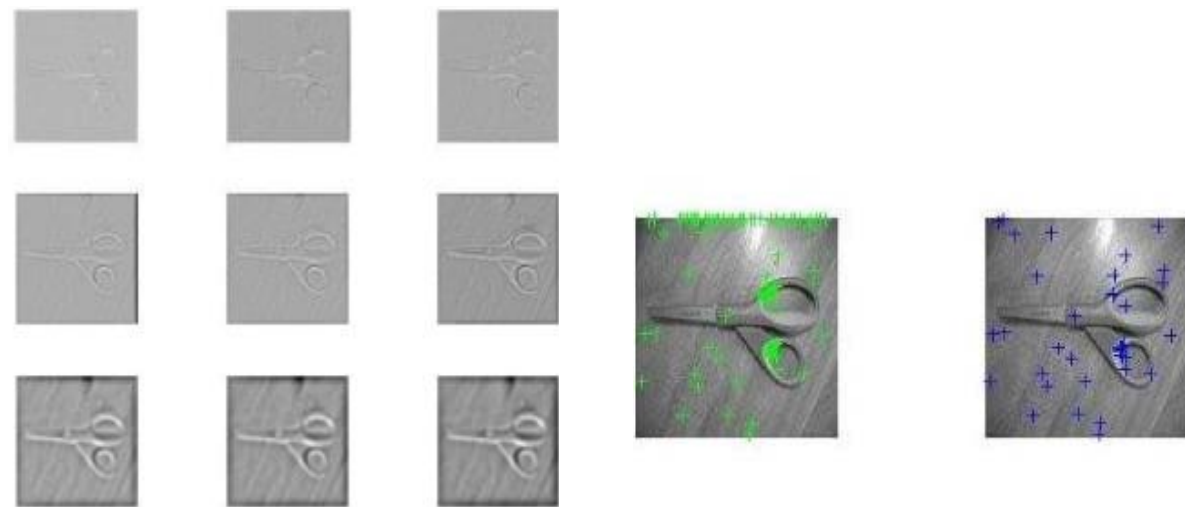

(a)

(b)

Figure 14 Industrial machine tool 1 results using SIFT (see online version for colours)
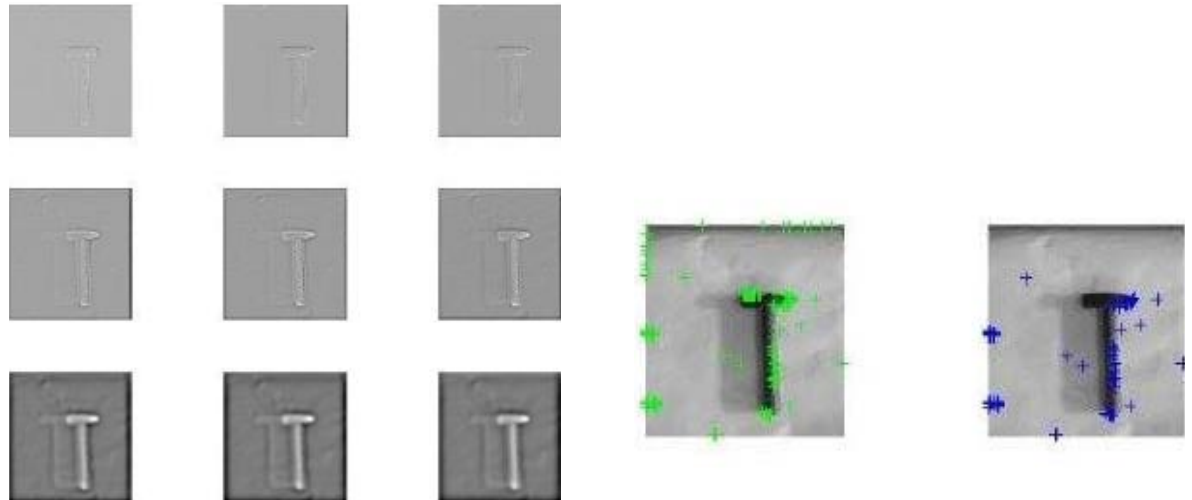

(a)

(b) 
Figure 15 Industrial CAD part 4 results using SIFT (see online version for colours)
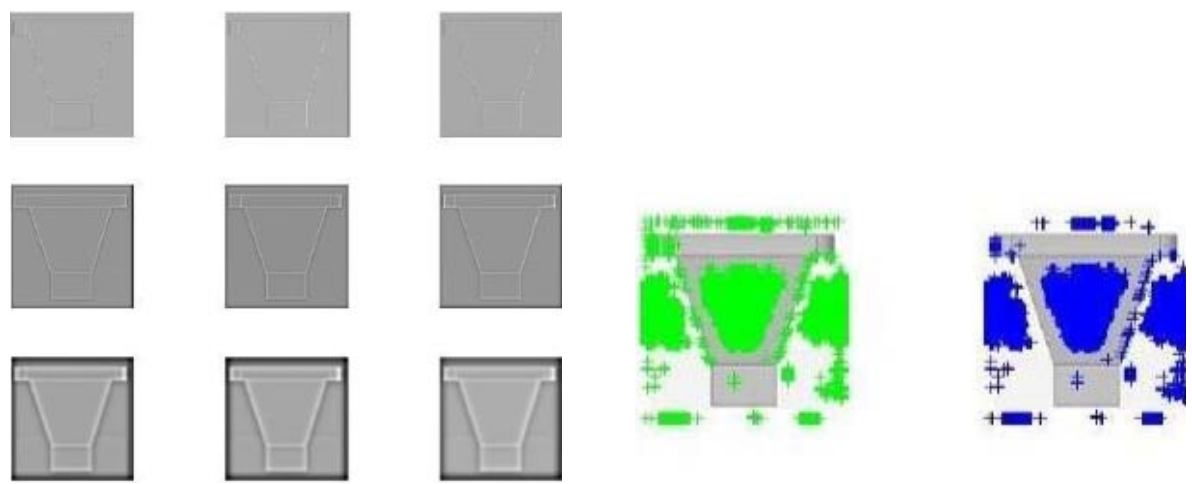

(a)

(b)

Figure 16 Industrial CAD part 5 results using MSER (see online version for colours)

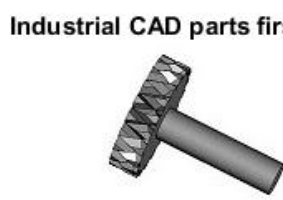

(a)

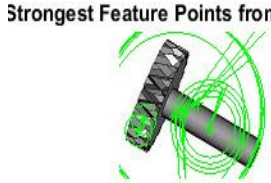

(b)

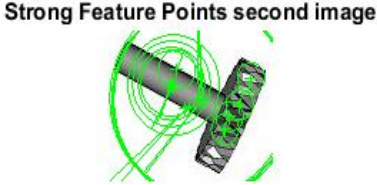

(c)

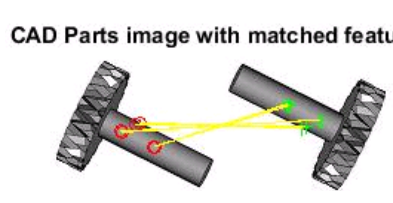

(d)

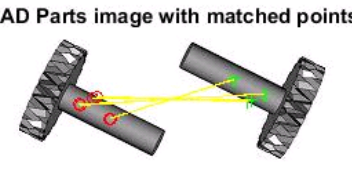

(e)

Industrial CAD Parts Detected

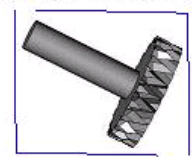

(f)

Figure 17 Industrial tools results using MSER (see online version for colours)

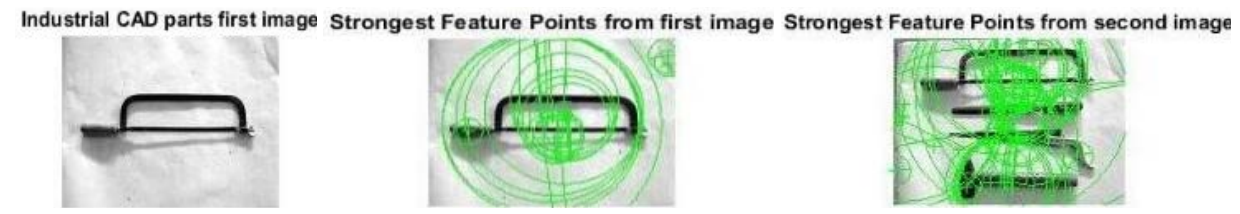

(a)

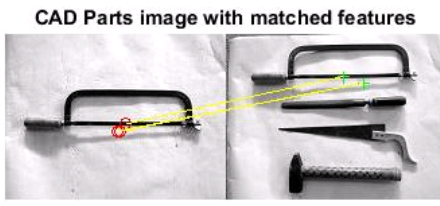

(d) (b)

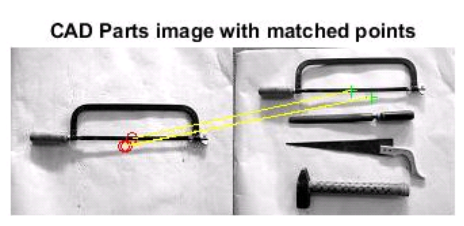

(e) (c)

Industrial CAD Parts Detected

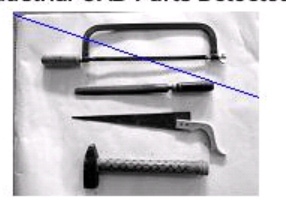

(f) 
Figure 18 Industrial tools results using MSER (see online version for colours)

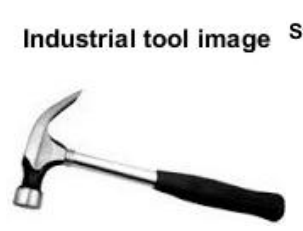

(a)

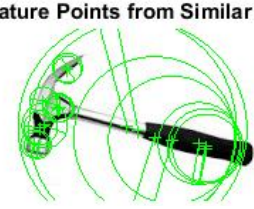

(b)

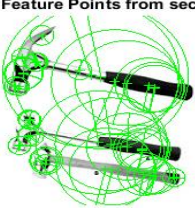

(c)

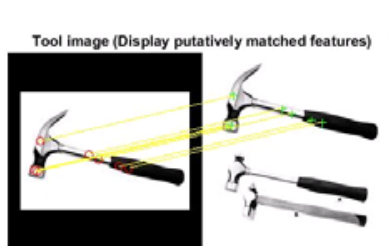

(d)

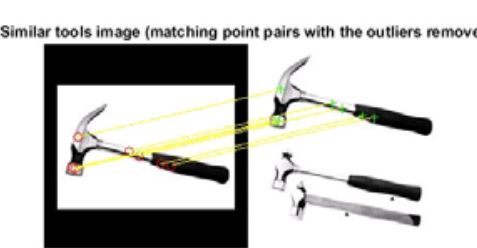

(e)

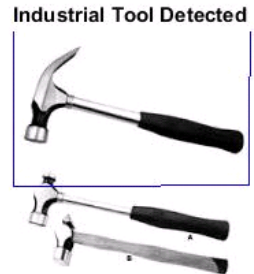

(f)

Figure 19 Industrial CAD parts results using MSER (see online version for colours)

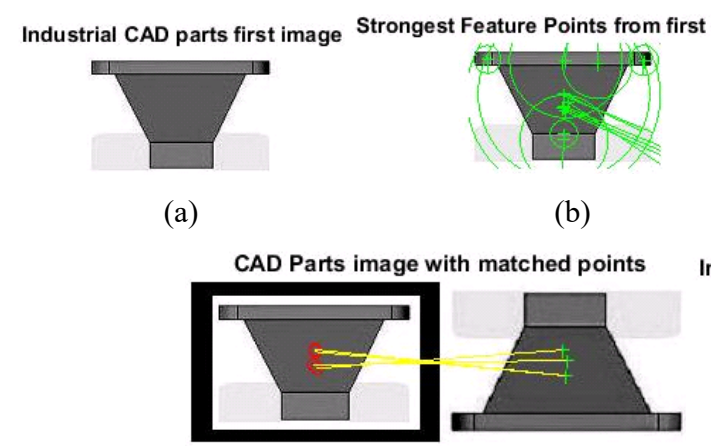

(d)

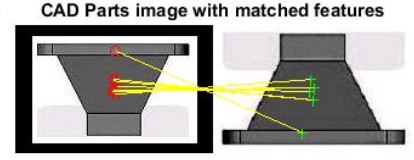

(c)

Industrial CAD Parts Detected

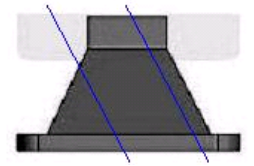

(e)

Figure 20 Industrial tools results using MSER (see online version for colours)

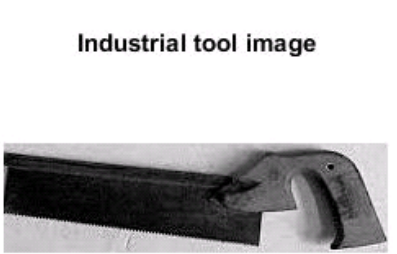

(a)

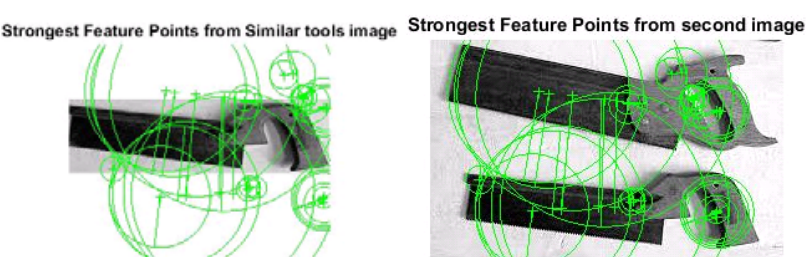

(b) (c)

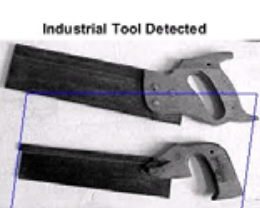

(f) 
Figure 21 Industrial tools results using SURF (see online version for colours)

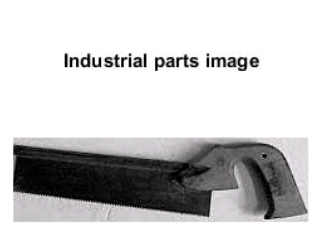

(a)

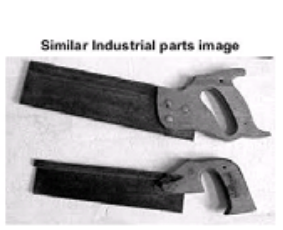

(b)

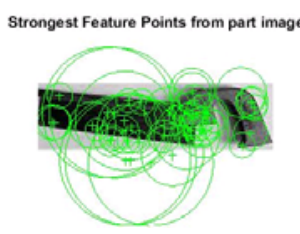

(c)

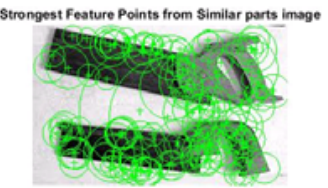

(d)

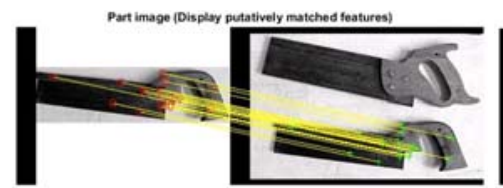

(e)

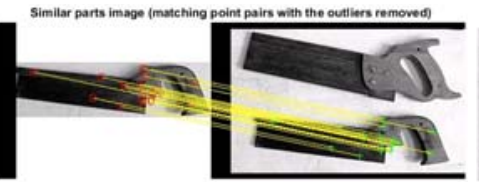

(f)

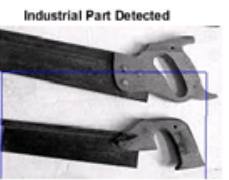

(g)

Figure 22 Industrial tools results using SURF (see online version for colours)

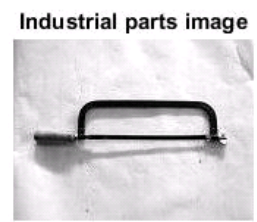

(a)

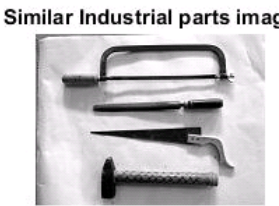

(b)

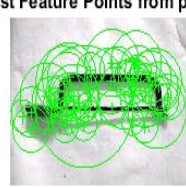

(c)

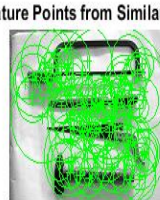

(d)

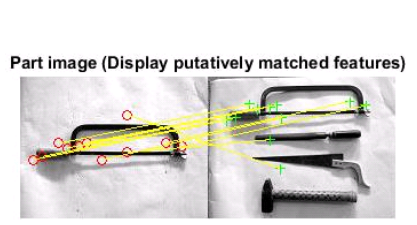

(e)

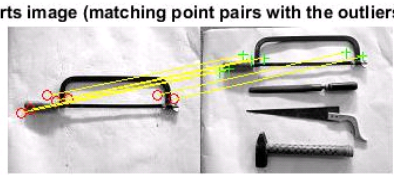

(f)

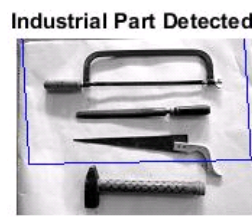

(g)

Figure 23 Industrial tools results using SURF (see online version for colours)

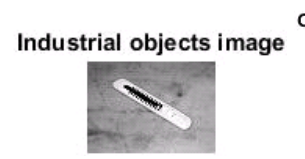

(a)

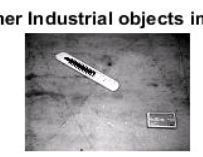

(b)

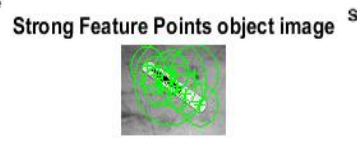

(c)

Strong Feature Points other object image

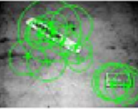

(d) Object image (Display putatively matched features)

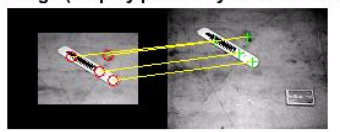

(e)
Similar objects image with the outliers removed

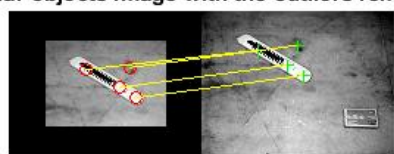

(f)

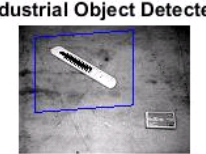

(g) 
Figure 21 represents the SURF results on saw tool. This method shows the better results as compared to other techniques on similar kind of objects. In the similar sequence Figure 22 shows the performance of this algorithm on variety of tools in the target image and one tool in the query image. The result finally gives the expected outcome. Figure 23 is another instance in which paper cutter tool is detected. The first image shows the 2D paper cutter tool object which is to be recognised with other industrial object image. The strongest features extracted from objects image are compared with the target image and the tool is detected. Figure 24 shows the same tool image detection using FAST method. As we compare the results of Figure 23 with Figure 24 the only difference is in the number of features extracted from the source as well as target object image in both the cases. SURF uses more features to detect a specific object and in general it can be used in a variety of practical applications whereas FAST does not give satisfactory results in those specific cases.

Figure 24 Industrial tools results using FAST (see online version for colours)

Industrial objects image other Industrial objects image

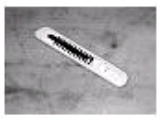

(a)

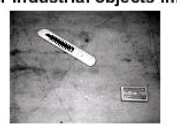

(b)

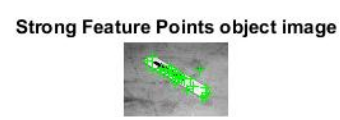

(c) ingest Feature Points from other objects in

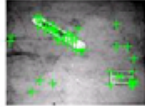

(d)

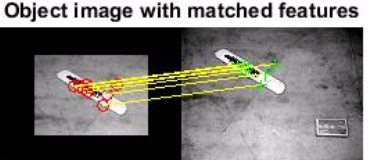

(e)

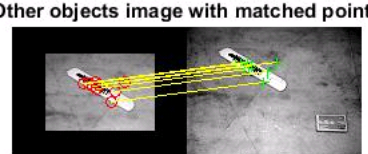

(f)

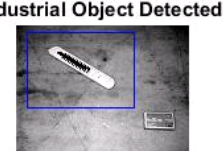

(g)

Figure 25 Industrial objects results using SURF (see online version for colours)

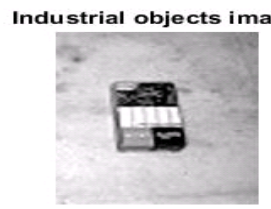

(a)

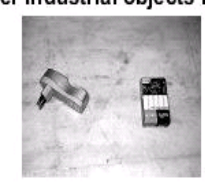

(b)

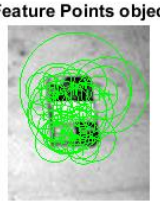

(c)

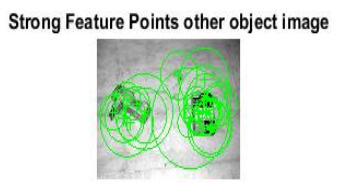

(d)

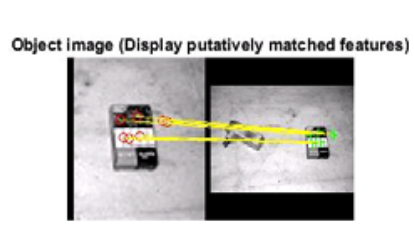

(e)

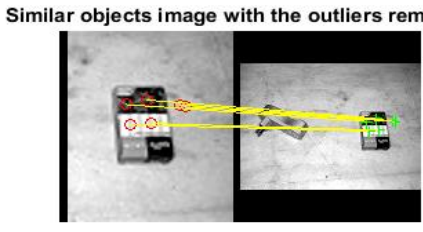

(f)

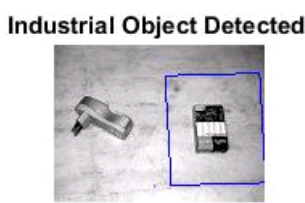

(g)

Finally, Figure 25 shows another instance of industrial object detection using SURF. If we compare this with previous results shown in different figures it is more realistic and practical. By this example we can conclude that out of varieties of feature-based 
techniques available for object recognition SURF can be used in different situations and on diverse objects whether $2 \mathrm{D}$ or 3D. The main distinction in these calculations depends on the component extraction. As we see BRISK calculation distinguish BRISK components and return BRISK Points protest similarly FAST method recognise corners and return cornerPoints question. For order assignments the HOG and SURF methods are utilised. While, BRISK and FREAK double descriptors calculations are particularly connected to discover point mapping between items pictures. Paired descriptors are regularly quicker yet with less exactness than inclination-based descriptors. For better exactness of protest acknowledgment we need to consolidate mutually extraordinary location and depiction methods as indicated Table 1 and Table 2.

Table 1 Selection of feature-based detector

\begin{tabular}{lcc}
\hline Detection algorithms & Types of feature category & Independency about scale \\
\hline SURF & Blob & Yes \\
BRISK & Corner & Yes \\
FAST & Corner & No \\
MSER & Region with uniform intensity & Yes \\
\hline
\end{tabular}

Table 2 Selection of description technique

\begin{tabular}{|c|c|c|c|c|c|c|}
\hline \multirow[b]{2}{*}{ S. no. } & \multirow{2}{*}{$\begin{array}{l}\text { Description } \\
\text { techniques }\end{array}$} & \multirow[b]{2}{*}{ Binarisation } & \multicolumn{2}{|c|}{ Invariant } & \multicolumn{2}{|c|}{ Special purpose } \\
\hline & & & Scaling & Rotating & $\begin{array}{c}\text { Matching } \\
\text { point locations }\end{array}$ & $\begin{array}{c}\text { Classifying } \\
\text { ability }\end{array}$ \\
\hline 1 & BRISK & Yes & Yes & Yes & Yes & No \\
\hline 2 & SURF & No & Yes & Yes & Yes & Yes \\
\hline 3 & FREAK & Yes & Yes & Yes & Yes & No \\
\hline 4 & HOG & No & No & No & No & Yes \\
\hline
\end{tabular}

\section{Conclusions and future work}

This technique shows a productive picture acknowledgment calculation for industrial CAD parts coordinating framework. SURF is utilised for removing highlights from reference parts and test images. A few employments of this framework are the programmed distinguishing proof of CAD parts on the web. This framework is utilised to distinguish both close copy parts and in addition parts with some fluctuation in their appearance. To think about the outcomes industrial CAD parts coordinating frameworks SIFT, FAST, BRISK, SURF, FREAK and MSER calculations are executed utilising MATLAB. The execution is assessed by changing the scale also the turn point. The investigation demonstrates that the proposed framework reliably beats other existing framework in parts coordinating and acknowledgment. For future work more questions either CAD parts or mechanical apparatuses can be taken for acknowledgment and recognition of deformities in them if any for mechanised examination reason. 


\section{References}

Alshennawy, A.A. (2014) 'Extract the geometry of mechanical parts by vision system using Hough transform', International Journal of Control, Automation and Systems, Vol. 3, No. 2, pp.9-14.

Bai, X., Yang, X. and Latecki, L.J. (2008) 'Detection and recognition of contour parts based on shape similarity’, Pattern Recognition, Vol. 41, No. 7, pp.2189-2199.

Bay, H., Tuytelaars, T. and Gool, L.V. (2006) 'SURF: speeded up robust features', Ninth European Conference on Computer Vision, Ser. LNCS, Springer-Verlag, Berlin, Vol. 3951, p.4040417.

Beserra Gomes, R., Ferreira da Silva, B.M., Rocha, L.K.D.M., Aroca, R.V. and Velho, L.C.P.R. (2013) 'Efficient 3D object recognition using foveated point clouds', Computers \& Graphics, Vol. 37, No. 5, pp.496-508.

Bicego, M., Castellani, U. and Murino, V. (2005) 'A hidden Markov model approach for appearance-based 3D object recognition', Pattern Recognition Letters, Vol. 26, No. 16, pp.2588-2599.

Chryssolouris, G. (2006) Manufacturing Systems: Theory and Practice, 2nd ed., Springer-Verlag, New York.

Farman, A., Khan, S.U., Mahmudi, M.Z. and Ullah, R. (2016) 'A comparison of FAST, SURF, Eigen, Harris, and MSER features', International Journal of Computer Engineering and Information Technology, Vol. 8, No. 6, pp.100-105.

Frome, A., Huber, D., Kolluri, R. and Thomas, B. (2004) 'Recognizing objects in range data using regional point descriptors', European Conference on Computer Vision, Vol. 1, pp.1-14.

Golnabi, H. and Asadpour, A. (2007) 'Design and application of industrial machine vision systems', Robotics and Computer-Integrated Manufacturing, Vol. 23, No. 6, pp.630-637.

Han, S.H., Choi, J.W., Son, K., Lee, M.C. and Lee, J.M. (2001) 'A study on feature-based visual servoing control of eight axes-dual arm robot by utilizing redundant feature', IEEE International Conference on Robotics and Automation, Vol. 3.

Han, S.H., See, W.H., Lee, J., Lee, M.H. and Hashimoto, H. (2000) 'Image-based visual servoing control of a SCARA type dual-arm robot', ISIE'2000. Proceedings of the 2000 IEEE International Symposium on Industrial Electronics, Vol. 2.

Harris, C. and Stephens, M. (1988) 'A combined corner and edge detection', Proceedings of the Fourth Alvey Vision Conference, pp.147-151.

Janocha, H.K. and Seidel, H. (1988) 'Image processing in a production environment', CIRP Annals - Manufacturing Technology, Vol. 37, No. 2, pp.579-590.

Kodagali, J. and Balaji, S. (2012) 'Computer vision and image analysis based techniques for automatic characterization of fruits - a review', International Journal of Computer Applications, Vol. 50, No. 6, pp.6-12.

Laptev, I. (2009) 'Improving object detection with boosted histograms', Image and Vision Computing, Vol. 27, No. 5, pp.535-544.

Li, C. and Ma, L. (2009) 'A new framework for feature descriptor based on SIFT', Pattern Recognition Letters, Vol. 30, No. 5, pp.544-557.

Lowe, D.G. (2004) 'Distinctive image features from scale-invariant keypoints', International Journal of Computer Vision, Vol. 60, No. 2, pp.91-110.

Matas, J., Chum, O., Urban, M. and Pajdla, T. (2002) 'Robust wide baseline stereo from maximally stable extremal regions', British Machine Vision Conference, pp.384-393.

Melkemi, M. and Djebali, M. (2001) 'Weighted a-shape: a descriptor of the shape of a point set', Pattern Recognition, Vol. 34, No. 6, pp.1159-1170.

Michalos, G., Makris, S., Eytan, A., Matthaiakis, S. and Chryssolouris, G. (2012) 'Robot path correction using stereo vision system', Procedia CIRP, pp.352-357.

Mikolajczyk, K. and Schmid, C. (2001) 'Indexing based on scale invariant interest points', Proceedings, ICCV, pp.525-531. 
Mikolajczyk, K. and Schmid, C. (2005) 'A performance evaluation of local descriptors', IEEE Transactions on Pattern Analysis and Machine Intelligence, Vol. 27, No. 10, pp.1615-1630.

Mikolajczyk, K., Tuytelaars, T., Schmid, C., Zisserman, A., Matas, J., Schaffalitzky, F., Kadir, T. and Gool, L.V. (2005) 'A comparison of affine region detectors', International Journal of Computer Vision, Vol. 65, No. 1, pp.43-72.

Moreels, P. and Perona, P. (2007) 'Evaluation of features detectors and descriptors based on 3D objects', International Journal of Computer Vision, Vol. 73, No. 3, pp.263-284.

Mortensen, E., Deng, H. and Shapiro, L. (2005) 'A SIFT descriptor with global context', Proceedings of IEEE Computer Society Conference on Computer Vision and Pattern Recognition (CVPR), San Diego, CA, USA, 20-26 June, pp.184-190.

Panchal, P.M., Panchal, S.R. and Shah, S.K. (2013) 'A comparison of SIFT and SURF', International Journal of Innovative Research in Computer and Communication Engineering, Vol. 1, No. 2, pp.323-327.

Pham, T.V. and Smeulders, A.W.M. (2005) 'Object recognition with uncertain geometry and uncertain part detection', Computer Vision and Image Under-Standing, Vol. 99, No. 2, pp.241-258.

Santochi, M. and Dini, G. (1998) 'Sensor technology in assembly systems', CIRP Annals Manufacturing Technology, Vol. 47, No. 2, pp.503-524.

Schmid, C., Mohr, R. and Bauckhage, C. (2000) 'Evaluation of interest point detectors', International Journal of Computer Vision, Vol. 37, No. 2, pp.151-172.

Scovanner, P., Ali, S. and Shah, M. (2007) 'A 3-dimensional SIFT descriptor and its application to action recognition', Proceedings of the 15th International Conference on Multimedia MULTIMEDI'07, Vol. 357.

Seib, V., Kusenbach, M., Thierfelder, S. and Paulus, D. (2014) 'Object recognition using houghtransform clustering of SURF features', Scientific Cooperations International Workshops on Electrical and Computer Engineering Subfields, Koc University, Istanbul/Turkey, pp.176-183.

Shokoufandeh, A., Marsic, I. and Dickinson, S.J. (1999) 'View-based object recognition using saliency maps', Image and Vision Computing, Vol. 17, No. 5, pp.445-460.

Tsarouchi, P., Matthaiakis, S., Michalos, G., Makris, S. and Chryssolouris, G. (2016) 'A method for detection of randomly placed objects for robotic handling', CIRP Journal of Manufacturing Science and Technology, Vol. 14, pp.20-27.

Vahrenkamp, N., Böge, C., Welke, K., Asfour, T. and Walter, J. (2009) 'Visual servoing for dual arm motions on a humanoid robot', 9th IEEE-RAS International Conference on Humanoid Robots, HUMANOIDS09, pp.208-214.

Xie, S.Q., Haemmerle, E., Cheng, Y. and Gamage, P. (2008) 'Vision-guided robot control for 3D object recognition and manipulation', in Ceccarelli, M. (Ed.), InTech, Croatia, Europe.

Zhao, J., Zhu, S. and Huang, X. (2013) 'Real-time traffic sign detection using SURF features on FPGA', IEEE, pp.323-327. 